\title{
Grandes personajes de la medicina, grandes lecciones
}

Enrique Ruelas-Barajas, ${ }^{1 *}$ Martha E. Rodríguez-Pérez ${ }^{2}$ y Ana C. Rodríguez de Romo ${ }^{2}$

${ }^{1}$ Universidad Panamericana, Escuela de Medicina; ${ }^{2}$ Universidad Nacional Autónoma de México, Facultad de Medicina, Departamento de Historia y Filosofía de la Medicina. Ciudad de México, México

\section{Resumen}

El análisis de tres personajes correspondientes a espacios y tiempos diferentes muestra el estrecho vínculo entre la literatura y la historia de la medicina. Por un lado, don Quijote de la Mancha, quien refleja el pensamiento de los últimos años del Renacimiento y ha sido asimilado en el México contemporáneo. Por otro lado, los doctores Miguel Francisco Jiménez y Rita Levi Montalcini, quienes vivieron en los siglos XIX y XX, respectivamente. A pesar de los años que separan a los tres personaje, se advierten numerosos elementos en común que no pierden vigencia: el valor que se otorga a la salud, la ética, la tenacidad y la experiencia para obtener resultados exitosos, entre otros. Los tres personajes aluden a la medicina de su tiempo, los logros alcanzados y la promoción del humanismo, siempre inherente a la medicina.

PALABRAS CLAVE: Medicina del Renacimiento. Medicina del siglo XIX. Premio Nobel de Medicina.

\section{Great personalities of medicine. Great lessons}

\begin{abstract}
The analysis of three characters corresponding to different spaces and times shows the close link between literature and the history of medicine. On one hand, Don Quixote of La Mancha, who reflects the thought of the last years of the Renaissance and that has been assimilated in contemporary Mexico. On the other hand, Doctors Miguel Francisco Jiménez and Rita Levi Montalcini who lived in the $19^{\text {th }}$ and $20^{\text {th }}$ centuries, respectively. Despite the years that separate these three personalities, many elements in common are observed that do not lose their validity: the value that is given to health, ethics, tenacity and experience to attain successful results. All three characters refer to the medicine of their time, their achievements and the promotion of humanism, always inherent to medicine.
\end{abstract}

KEY WORDS: Medicine of the Renaissance. $19^{\text {th }}$ century medicine. Nobel Prize in Medicine.

\section{Introducción}

La cultura, como un fenómeno social que da profundidad a nuestra especie, es una dimensión que debe caracterizar al médico más que a muchos otros cuya profesión no tiene el carácter humano de la medicina. En estos tiempos difíciles de pandemia, la cultura se convierte también en un bálsamo para paliar el estrés y la incertidumbre. Es posible atestiguarlo mediante la enorme cantidad de mensajes que circulan por las redes sociales con piezas de música, clásica y del momento, imágenes de pinturas y textos literarios e históricos con objeto de tranquilizar los espíritus atribulados por la situación y de fortalecer a quienes, afortunados, pueden disfrutar de algo enriquecedor entre las paredes del encierro.
Correspondencia:

*Enrique Ruelas-Barajas

E-mail: eruelas@ @rodigy.net.mx
Fecha de recepción: 14-05-2020

Fecha de aceptación: 26-05-2020

DOI: 10.24875/GMM.20000284
Gac Med Mex. 2020;156:474-481

Disponible en PubMed

www.gacetamedicademexico.com

0016-3813/@ 2020 Academia Nacional de Medicina de México, A.C. Publicado por Permanyer. Este es un artículo open access bajo la licencia CC BY-NC-ND (http://creativecommons.org/licenses/by-nc-nd/4.0/). 
Cuando la doctora Teresita Corona, presidenta de la Academia Nacional de Medicina de México, propuso este tema para incluirlo en el programa de sesiones ordinarias, nadie podía sospechar que esta sesión fuese a coincidir no solo con una pandemia, sino con su momento más álgido. La diseminación de la cultura, desde la Academia Nacional de Medicina de México, cumple cabalmente sus propósitos, para los médicos y los no médicos.

En este documento se abordan tres personajes de tres momentos históricos en contextos muy diferentes. Transitaremos desde los últimos años del Renacimiento hasta el siglo XX, pasando por el siglo XIX. El primero de ellos es don Quijote de la Mancha, tema desarrollado por Enrique Ruelas; el segundo personaje de esta cronología es el doctor Miguel Francisco Jiménez, presentado por Martha Eugenia Rodríguez Pérez; y la tercera, es una mujer excepcional cuyos rasgos describe Ana Cecilia Rodríguez de Romo.

Don Quijote de la Mancha es un referente riquísimo de la medicina del Renacimiento a través de la enorme cantidad de citas sobre remedios, intervenciones y consideraciones en torno a los médicos y el ejercicio de su profesión que hace Miguel de Cervantes a través de sus personajes, principalmente don Quijote y Sancho Panza. De esta manera, su autor y su principal protagonista se convierten, como si fuesen uno, en un personaje de la medicina renacentista.

A continuación, se presenta el desarrollo profesional de un médico mexicano, Miguel Francisco Jiménez (1813-1875), quien perteneció a la élite académica del siglo XIX. Se abordan varias facetas, su desempeño docente al interior de la Escuela Nacional de Medicina, su actuar en asociaciones académicas, entre ellas la Sección Médica de la Comisión Científica, considerada el antecedente de la actual Academia Nacional de Medicina de México, donde formó parte de la mesa directiva. Por último, nos enfocamos en el trabajo clínico de Jiménez, cuyas contribuciones radican, entre muchas otras, en diferenciar el tifo de la fiebre tifoidea y en proponer un recurso terapéutico para el absceso hepático.

Finalmente, el descubrimiento del factor nervioso de crecimiento (FNC) y la vida de su descubridora, la médica italiana Rita Levi-Montalcini, constituyen historias fascinantes que ensamblan de forma maravillosa lo rico y productivo que pueden ser la vida y la obra de un ser humano. En este trabajo se abordará someramente a la investigadora obsesionada con su ciencia y a su descubrimiento, el cual aclaró el origen y desarrollo del tejido nervioso y la hizo merecedora del Premio Nobel.

\section{Medicina del Renacimiento en Don Quijote de la Mancha}

"Maestro: dígame cuál es el libro de medicina que condense todo el saber, que me haga comprender el dolor, el sufrimiento y las alegrías del hombre. Dígame, profesor para ello ¿qué libro debo leer?, ¿de qué autor?" Estas preguntas las hizo un alumno a Paul Ehrlich, médico alemán descubridor de la arsfenamina (salvarsán), la cual permitió dar tratamiento efectivo a la sífilis y por la que se le otorgó el Premio Nobel de Medicina en 1908. La respuesta de Ehrlich fue: "Lee el Quijote, hijo, de Miguel de Cervantes".

\section{Don Quijote: personaje de ficción y realidad}

¿Es posible considerar a don Quijote de la Mancha como personaje de la medicina? ${ }^{1}$ La respuesta es doblemente sí. Empecemos por argumentar la naturaleza de don Quijote como personaje y después como personaje de la medicina. En el Diccionario de la lengua española se define como personaje a "cada uno de los seres reales o imaginarios que figuran en una obra literaria, teatral o cinematográfica". Don Quijote obviamente lo es. Sin embargo, otra acepción de la palabra es "persona singular que destaca por su forma peculiar de ser o de actuar". A través de cuatro siglos de presencia ficticia, don Quijote es para muchos un personaje real, es alguien que existió; para ellos, don Quijote cumple también con la segunda definición.

A manera de evidencia de esta segunda acepción cito el fenómeno cultural que viera su nacimiento en el Guanajuato de mediados del siglo XX. En 1953, en la plaza de San Roque de la ciudad de Guanajuato, se escenificó por primera vez la obra Entremeses de Cervantes. Mundo imaginario y realidad de su mundo. La puesta en escena tuvo una enorme trascendencia con repercusiones hasta nuestros días, tanto por el escenario a cielo abierto en la explanada de un templo con una cruz en el centro, sobre un pedestal y rodeada de faroles torcidos que distribuía el espacio escénico, como por el montaje singular del espectáculo, que realizó Enrique Ruelas Espinosa, su creador y director. La obra se ha representado en ese espacio durante 67 años, a pesar del fallecimiento de su iniciador hace 33. Para celebrar 20 años de funciones ininterrumpidas, en 1972 se decidió realizar un Festival Internacional Cervantino, ahora también un evento de la mayor categoría cultural en el mundo, 
que ha llegado a 47 años de vida. Esto, y otros importantes acontecimientos que se desencadenaron en torno a Miguel de Cervantes y su obra en esa ciudad, como la creación del Museo Iconográfico del Quijote, motivó a la UNESCO a declarar en 2005 a Guanajuato como "capital cervantina de América". Por tal motivo, Eulalio Ferrer, distinguido hombre de letras y gran cervantista, donó una escultura de don Quijote para señalar este acontecimiento, la cual fue colocada sobre un pedestal en el centro de la ciudad; el sitio donde se ubica es visitado por los turistas, a quienes algunos guías indican que se trata de la tumba donde se encuentran enterrados los restos de don Quijote. Y algunos lo creen.

En 2010, para aprovechar esta creencia y al mismo tiempo hacer evidente la vida ficticia, aunque casi real del famosísimo personaje, la Fundación Cervantista "Enrique y Alicia Ruelas" y el Museo Iconográfico del Quijote decidieron "enterrar" a don Quijote de la Mancha en ese mismo lugar, por supuesto, en su versión editorial. La placa que señala el lugar dice así:

\begin{abstract}
Ha muchos años llegó a Guanajuato el Caballero de la Triste Figura en áurea cabalgadura de teatral imaginación, tenaz bonhomía y enorme entusiasmo de Enrique Ruelas, de Eulalio Ferrer y de muchos guanajuatenses. En la base de su escultura, pero no a manera de sepultura, yace la Edición Guanajuato de Don Quijote de la Mancha. Quien afirme que Don Quijote está en esta tierra enterrado, jamás mentirá. En la Ciudad de Guanajuato, sus pobladores lo enterraron en este lugar como a un colosal y transparente tesoro para gloria de Miguel de Cervantes Saavedra, del espíritu humano y de la cultura universal.
\end{abstract}

De esta manera, don Quijote, el personaje ficticio, se funde con el personaje real y, para muchos, con su autor, personaje de la literatura universal. Así, para hablar de don Quijote de la Mancha como personaje de la medicina es menester hablar del personaje real que lo creó: Miguel de Cervantes Saavedra (Figura 1).

\section{Personajes de la medicina del Renacimiento}

Cervantes nació en Alcalá de Henares en 1547 y falleció en 1616. Vivió justamente en la etapa final del Renacimiento, si se considera que el tiempo arbitrariamente asignado a este periodo va de 1450 a 1600, que para algunos se extiende hasta 1616, precisamente el año del fallecimiento de Cervantes y, casi en la misma fecha, también de William Shakespeare. Coincidió en el tiempo con forjadores no solo de este fascinante

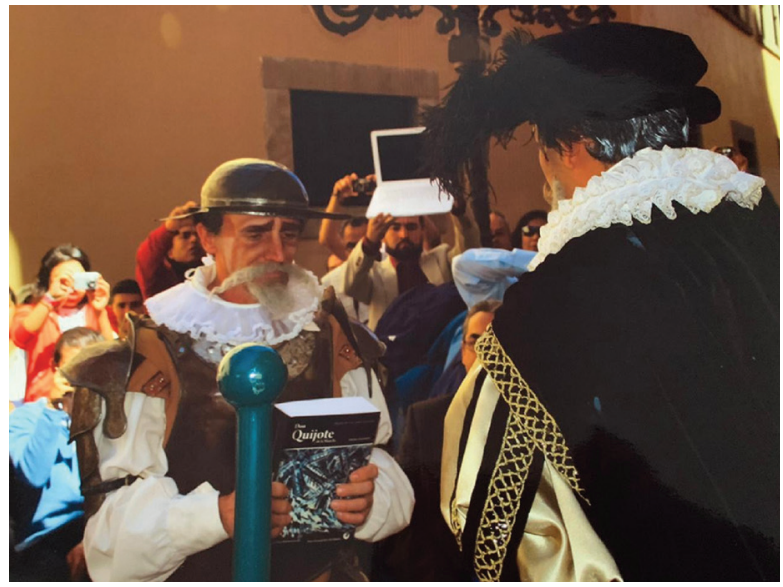

Figura 1. El entierro del Quijote en Guanajuato.

periodo, sino del arte y de la ciencia de nuestros días. Contemporáneos de Cervantes fueron Lope de Vega, Quevedo, Miguel Ángel Buonarroti, Velázquez, el Greco, Vesalio, Harvey, Bacon, Leeuwenhoek, Hook, Paré, Descartes, Galileo y Kepler. De esta manera, Cervantes transitó a través de revoluciones en la anatomía, fisiología, cirugía, microscopia y en la clínica. Fue un digno ciudadano del Renacimiento. Además, su padre fue médico, por lo que seguramente recibió su influencia y ciertos conocimientos que tejió finamente en su obra. A pesar de haber sido soldado y recaudador de impuestos, Cervantes fue un hombre culto.

Por ello, don Quijote de la Mancha, como libro y ficción de un entrañable caballero andante, es un personaje no solo de la literatura sino también de la medicina. Don Quijote se define como médico de alguna manera cuando dice: "el caballero andante ha de ser médico y principalmente herbolario para conocer en mitad de los despoblados y desiertos en que se encuentre, las hierbas que tienen virtud de sanar feridas." En el texto se mencionan 281 términos médicos que aparecen en 4226 ocasiones. El vocablo más citado es "sangre". En los diferentes personajes de la obra aparecen signos dermatológicos, infecciosos, neurológicos, oftalmológicos y urinarios, entre otros. Se habla de heridas por todas partes. Dice don Quijote: "Las feridas que se reciben en las batallas antes dan honra que la quitan" y "...si no me quejo del dolor es porque no es dado a los caballeros andantes quejarse de ferida alguna, aunque se le salgan las tripas por ella".

Remedios e intervenciones también están presentes a lo largo de la obra. Se recomiendan plantas como achicoria, adelfa, romero, ruibarbo, tártago y verbena; 
- el famoso bálsamo de Fierabrás. Se prescriben ungüentos como el de altea, basilicón y blanco. Las recetas contienen nombres, modo de prescripción e instrucciones de uso. Se practican intervenciones como fuentes, sedales, melecinas y diferentes tipos de sangrías. Sancho Panza, como acostumbra, pronuncia sobre los médicos, entre otras, una cómica frase: "más quiero hartarme de gazpacho que estar sujeto a la miseria de un médico impertinente que me mate de hambre"; y una iluminada sentencia cuando ha sido investido gobernador de la Ínsula Barataria: "no me ha de quedar médico en toda la Ínsula a lo menos de aquellos que son ignorantes, que a los médicos sabios, prudentes y discretos les pondré sobre mi cabeza y los honraré como a personas divinas".

No queda duda, don Quijote de la Mancha, como obra literaria, como personaje de ficción casi real y como expresión de un renacentista cabal, es un personaje de la medicina. Ambos lo son: don Quijote y Miguel de Cervantes. En efecto, como dijo Ehrlich a su alumno, para saber medicina hay que acercarse al Quijote de Miguel de Cervantes.

\section{Miguel Francisco Jiménez y la clínica del siglo XIX}

Miguel Francisco Jiménez (1813-1875) nació en el estado de Puebla, fue un hombre multifacético con gran conciencia histórica, de ahí que podamos tener información de su propia mano acerca de sus actividades como catedrático, integrante de asociaciones académicas, editor y clínico. Su desempeño profesional transcurrió a través de los diferentes hechos que vivía el país, la dictadura de Santa Anna, la invasión norteamericana, la intervención francesa, durante la cual apoyó al Segundo Imperio. Jiménez creyó en el gobierno de Maximiliano, esperanzado de que daría estabilidad sociopolítica y cultura al país. Así lo hizo saber al ministro de hacienda José María Iglesias: “...tengo fe de que podría fundarse un orden que, realmente aceptado por todos, acabaría para siempre con la eterna anarquía que nos consume". 2 De hecho, fue uno de los cuatro médicos personales que tuvo el emperador, además de Federico Semeleder, Samuel Basch y Rafael Lucio.

\section{El actuar de Miguel Francisco Jiménez en la Escuela de Medicina}

Jiménez se tituló de médico cirujano en septiembre de 1838 y dos meses después empezó a trabajar como profesor en la Escuela de Medicina de la Ciudad de México. Impartió cuatro materias: Anatomía, Prosector de Anatomía, Patología Interna y Clínica Interna. Asimismo, se desempeñó como secretario de la institución, de la cual llegó a conocer bien su funcionamiento.

Como profesor de Clínica Interna mostró múltiples habilidades. Publicó sus lecciones en la prensa especializada, enseñó a sus alumnos el método para hacer un diagnóstico: inspección, palpación, percusión y auscultación, como a su vez Jiménez lo aprendió de su maestro de fisiología, Manuel Carpio. En la inauguración de esa asignatura, Jiménez, apoyado en Bouillard, afirmó que "el método es, sin disputa, el supremo regulador de todas las cosas...., ${ }^{3}$ de ahí que llegara a ser pionero en el tratamiento de los abscesos hepáticos.

\section{Asociaciones académicas}

En el marco de la intervención francesa, en 1864 se instituyó la Comisión Científica, Literaria y Artística de México, integrada por varias secciones, entre ellas la sexta, la de Ciencias Médicas, cuyos dirigentes fueron Carlos Alberto Ehrmann (presidente), Julio Clement (primer vicepresidente) y Miguel F. Jiménez (segundo vicepresidente). Tras el retiro hacia Europa del comando francés y del mismo Ehrmann, la sección médica se independizó en 1865 y se denominó Sociedad Médica de México, bajo la presidencia de Jiménez, Sociedad que en 1870 cambiaría su nombre por el de Academia de Medicina de México. De manera que este organismo tuvo como primer presidente a Ehrmann y como segundo, a Jiménez, quien ocupó el cargo en cuatro ocasiones: en 1865, 1866, 1870 y 1872, dado su profundo interés por la ciencia. Asimismo, en el marco de esta agrupación, Jiménez se desempeñó como presidente de la Comisión de Publicaciones, que editó Gaceta Médica de México a partir del 15 de septiembre de 1864.

En 1870, cuando Jiménez fungió nuevamente como titular de la asociación, externó su interés por la situación del país, proponiendo que cada socio presentara por turno un trabajo original en las sesiones de los miércoles, donde se analizaran los problemas de salud en el país ${ }^{4}$ (Figura 2).

\section{Jiménez, clínico por excelencia}

Miguel Francisco Jiménez fue un clínico talentoso. Tuvo grandes maestros. En el ámbito internacional 


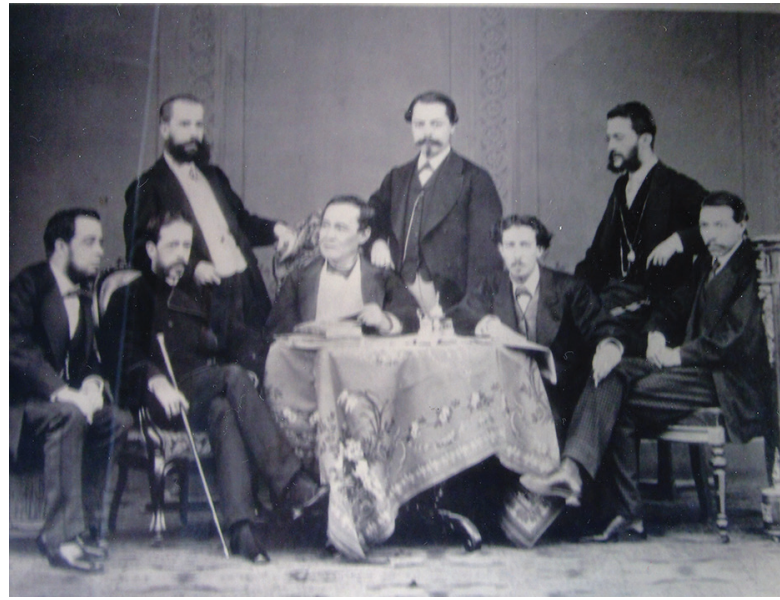

Figura 2. Miguel F. Jiménez, sentado en el centro, en la Sociedad Familiar de Medicina, 1870.

podríamos decir que siguió cuidadosamente los avances de la escuela francesa, que tuvo el mérito de haber creado y enseñado la mentalidad anatomoclínica, en la cual lo fundamental en la enfermedad es la lesión anatómica y el examen de las alteraciones de los órganos, como lo plantearon Corvisart y Laennec, entre otros. Jiménez estudió particularmente las lecciones de Magendi, pionero de la fisiología experimental. ${ }^{5}$ En cuanto a sus maestros nacionales, Jiménez fue discípulo de Manuel Carpio, quien introdujo en México los saberes y aportaciones de Laennec, como la auscultación, el acercamiento a las enfermedades cardiacas y el estetoscopio.

Al auscultar y usar el estetoscopio, Jiménez pudo conocer lo que sucedía en el interior del cuerpo humano, lo que parecía haber estado encubierto. Así, expresó: "merced a los descubrimientos inmortales de Aüembrugger y de Laennec, han dado al médico la facultad de ver hasta el interior de los órganos como si el cuerpo humano fuese transparente..."3

Particularmente se presentan dos aportaciones de Miguel F. Jiménez al área clínica, la diferencia entre el tifo y la fiebre tifoidea y, por otra parte, un recurso terapéutico para el absceso hepático amebiano.

En cuanto a la primera aportación, Jiménez estudió el tifo o tabardillo durante muchos años, ya que fue una enfermedad asociada a la pobreza e insalubridad presentes en México. Al consultar los libros de autores franceses que hacían referencia a la fiebre tifoidea, Jiménez esclareció dudas. Se entrelazaban ambas enfermedades porque el término fiebre era confuso. De tiempo atrás procedía el error de convertir un signo clínico en entidad patológica, ${ }^{6}$ consistente, entre otras cosas, en un complejo de alteraciones como estupor, cuadros disneicos, dolor y diarrea, además del aumento de calor en el cuerpo del enfermo; Jiménez logró dilucidar semejanzas y diferencias entre el tifo y la fiebre tifoidea. Al respecto señaló: "creo tener el honor de haber sido el primero que llamara la atención sobre las diferencias que se notan entre el tabardillo de México y la fiebre de Europa..."

En cuanto al absceso hepático, Jiménez lo estudió porque representaba una enfermedad habitual en el país. Durante 18 años atendió a los enfermos en el Hospital de San Andrés, transformado en un espacio de emergencia de prácticas científicas de carácter público. ${ }^{8}$ Tuvo el cuidado de dejar por escrito sus observaciones a manera de lecciones de clínica en el periódico La Unión Médica de México. Al respecto expresó: ${ }^{9}$

\footnotetext{
...pues bien, un líquido que se ha desarrollado en el hígado después de una inflamación aguda, con las circunstancias que van referidas, debe ser pus; y de consiguiente la enfermedad que hoy estudiamos debe calificarse así: abscesos del hígado.
}

Después de analizar numerosos casos clínicos de pacientes con absceso hepático, Jiménez planteó su hipótesis respecto a la etiología. Propuso que las enfermedades hepáticas eran más frecuentes en zonas cálidas, como México, que en climas fríos. ${ }^{9} \mathrm{El}$ sexo y grupo etario predominante era de hombres entre 28 y 58 años y como antecedentes del padecimiento encontró indigestión grave, actividad física intensa, episodios de enojo y, fundamentalmente, alcoholismo, refiriéndose en particular al consumo de pulque fermentado.

Ya identificado el padecimiento, Jiménez propuso un recurso terapéutico: el drenaje artificial a través de la punción por trócares, con lo que logró disminuir la mortalidad por abscesos en $60 \%$. Gracias a su forma de trabajar bajo una rigurosa metodología, Jiménez se posicionó como gran clínico que trascendió las fronteras mexicanas.

\section{Rita Levi-Montalcini: elogio de la imperfección}

Rita Levi-Montalcini recibió el Premio Nobel de Medicina en 1986 por haber descubierto el FNC. Por difícil que parezca, a mediados del siglo XX se ignoraba mucho acerca del desarrollo del sistema nervioso; cómo se diferencian las células nerviosas entre ellas, cómo sus axones establecen las sinapsis o cuál es la naturaleza de los mensajes químicos con los que 
se comunican. El descubrimiento del FNC contestó esas preguntas y sigue contestando otras, ya que se han encontrado evidencias de su relación con la etiología de enfermedades como las de Alzheimer, Huntington, Parkinson y esclerosis múltiple.

\section{Cómo y por qué del interés por Rita Levi-Montalcini}

Hace algunos años llegó a mis manos la autobiografía de Rita Levi-Montalcini, titulada Elogio de la imperfección..$^{10}$ Rita era una excelente escritora y la lectura fresca y encantadora del libro me atrapó desde las primeras páginas. Después, como historiadora de la medicina, una de mis líneas de interés fue el estudio de los factores que rodean al fenómeno del descubrimiento científico: las circunstancias sociales, políticas, económicas e históricas, y, obviamente, la figura del mismo investigador, su personalidad, biografía, contexto, etcétera. El personaje de Rita LeviMontalcini y el FNC ocuparon por un buen tiempo mi interés y atención e, incluso, el ejemplo constituye un capítulo de mi libro ¿Eres exitoso? La historia y los científicos responden.11

Rita Levi-Montalcini nació el 22 de abril de 1909 en Turín, Italia, en el seno de una familia judía muy cultivada, y murió el 30 de diciembre de 2012 en Roma, a la edad de 103 años. Hasta el final conservó la lucidez, aunque estaba casi ciega y sorda. En esos últimos años solía decir: "es inevitable que el cuerpo se me arrugue, pero no el cerebro. Mantén tu cerebro ilusionado, activo, hazlo funcionar y nunca se degenerará."

Rita recibió la influencia de un padre vanguardista, pero cuidadoso de mantener las tradiciones familiares, y de una madre cultivada, pero obediente de los roles de poder en el matrimonio; ambos eran cultos y brillantes intelectualmente. Tuvo cuatro hermanos, pero su gemela Paola (1909-2000) ocupó un lugar especial en su vida y, además, fue su mejor amiga. De hecho, a ella dedicó su autobiografía.

Entre sus biógrafos es conocida su decisión de no casarse con la finalidad de dedicarse por completo a la ciencia. En efecto, Rita nunca se casó, pero siendo muy joven tuvo un pretendiente, también estudiante de medicina, a quien quiso considerablemente, pero quien falleció. Es difícil saber qué hubiera sucedido realmente si ese joven no hubiera muerto. La opinión de Rita LeviMontalcini para escoger pareja es muy atractiva; según ella, para decidir compartir la vida con alguien es fundamental considerar la diferencia de temperamento, la educación y los intereses culturales.

Respecto a Rita y Paola, su padre había decidido enviarlas a una escuela femenina donde aprenderían a ser buenas esposas y madres. Sin embargo, a los 20 años, Rita manifestó su deseo de estudiar medicina. Su padre se mostró renuente, pero la apoyó; en 1930, Levi-Montalcini entró a la Escuela de Medicina de Turín, donde entonces había solo siete mujeres entre 300 hombres. Su hermana Paola también se reveló al designio de su padre, se dedicó a la pintura y llegó a ser una artista de renombre; tampoco se casó.

\section{Trayectoria científica}

En 1932, cuando cursaba el segundo año de los estudios médicos, Rita ingresó al laboratorio del histólogo Giuseppe Levi (1872-1965), quien le enseñó la clásica tinción con sales de plata ideada por Santiago Ramón y Cajal (1852-1934), la cual usaría toda su vida. Con Levi conoció a sus amigos de siempre, Salvador Luria (1912-1991) y Renato Dulbecco (19142012). Años después, los tres colegas serían acreedores al Premio Nobel: Luria por aclarar el mecanismo de réplica y la estructura genética de los virus y Dulbecco, por descubrir la interacción de los virus tumorales y el material genético de las células. Las genealogías científicas son muy atractivas: en el caso de Levi-Montalcini, se iniciaría con Ramón y Cajal, ella a su vez fue alumna de Levi y su disciplina de estudio se continuaría con sus alumnos, actualmente dispersos en el mundo científico.

Al terminar la carrera en 1936, Levi-Montalcini pensó especializarse en neurología y psiquiatría, lo que no fue posible porque el Manifiesto en defensa de la raza de Benito Mussolini prohibió la presencia de los judíos en las universidades. Entonces, con Giuseppe Levi y en la clandestinidad, continuó sus investigaciones del sistema nervioso en un improvisado laboratorio casero, que ella describió como "al estilo Robinson Crusoe". Con su maestro Levi publicó algunos trabajos: uno en particular, de 1946, llamó la atención de Viktor Hamburger (1900-2001), científico alemán, estudioso de la embriología de esa época. El artículo abordaba el efecto de la extirpación en embriones de pollo, de las yemas que dan lugar al desarrollo de sus miembros. Hamburger escribió a Levi para invitar a su alumna a repetir ese experimento en su laboratorio de Saint Louis Missouri, en Estados Unidos. Rita LeviMontalcini llegó al laboratorio de Hamburger en 1947 


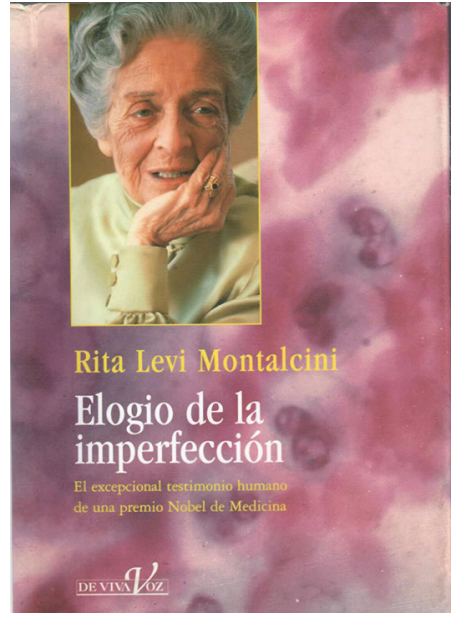

Figura 3. Portada del libro de Rita Levi-Montalcini.

y regresó definitivamente a su país ya siendo muy anciana.

En Roma fundó un instituto de investigación, se dedicó a apoyar a la ciencia, recibió múltiples distinciones y honores, fue senadora vitalicia, embajadora de Italia frente a la FAO (Organización de las Naciones Unidas para la Alimentación y la Agricultura), desarrolló una gran labor filantrópica en Etiopía y con sus ahorros impulsó la educación de las mujeres en los países subdesarrollados. No solo escribió artículos científicos, también redactó piezas literarias muy exitosas que, además de amenas, enseñan lecciones de vida. Ya mencioné su autobiografía Elogio de la imperfección (Figura 3). Otros libros son El as en la manga, La galaxia mente, Sin aceite y contra el viento y Teniendo el valor de saber, entre otros.

La saga del descubrimiento del FNC es un abanico de agudas observaciones, casualidades, sucesos fortuitos, golpes de suerte y factores circunstanciales magníficamente aprovechados por una científica genuina. En esta presentación no abordaré los detalles del descubrimiento de Rita Levi-Montalcini, lo que sería motivo de otro texto; ${ }^{12}$ más bien deseo que permeé el mensaje de la científica genuina, excepcional, que solo vivió para su ciencia.

\section{Premio Nobel}

En 1986, a la edad de 77 años, Rita Levi-Montalcini recibió el Premio Nobel por haber descubierto el FNC. Continúa causando controversia el hecho de que el Nobel no se haya otorgado también a Viktor Hamburger, puesto que fue en su laboratorio donde
Levi-Montalcini hizo los experimentos. Personalmente tengo múltiples explicaciones, pero cuando a la misma científica le preguntaron la razón contestó: "Viktor era un erudito, que siempre hizo un trabajo excelente, pero él nunca descubrió el factor nervioso de crecimiento". EI FNC fue el motivo de vida de Rita Levi-Montalcini. La manera como se refiere a él en la recepción del Premio Nobel es tan elocuente y extraordinaria, que se califica como la más bella pieza literaria que jamás se haya escrito respecto a la distinción con que sueñan todos los científicos. En su autobiografía narra lo siguiente:10

En la víspera de la Navidad de 1986, el factor nervioso de crecimiento se presentó al público a la luz de los reflectores, en el esplendor de una inmensa sala festivamente adornada en presencia de los Reyes de Suecia, de príncipes, de damas con ricos vestidos de gala y caballeros de esmoquin. Envuelto en un abrigo negro se inclinó ante el rey y por un instante apartó el velo que le cubría el rostro. Nos reconocimos inmediatamente, cuando vi que su mirada me buscaba entre la multitud que aplaudía. Luego volvió a cubrirse la cara y desapareció con la misma rapidez con que había llegado. ¿Habrá vuelto a su vida errante en los bosques habitados por los espíritus que vagan de noche por las orillas de los lagos helados del norte, donde pasé tantas horas solitarias y encantadas de mi juventud? ¿Volveremos a vernos algún día? ¿O fue aquel instante el cumplimiento del deseo de encontrarnos que había albergado durante tantos años y le habré perdido definitivamente de vista?

Rita Levi-Montalcini y el FNC significaban una unidad, un solo ser, eran siameses imposibles de separar a riesgo de que cualquiera de los dos muriera. El hallazgo de esta investigadora excepcional dio lugar a la comprensión de procesos básicos de la naturaleza del cuerpo humano. La destrucción o baja producción del FNC es causa de enfermedades nerviosas, algunas incurables. Levi-Montalcini probó que el FNC estimula las dos líneas celulares periféricas de la médula, motoras y sensitivas, y también las neuronas implicadas en funciones cerebrales superiores. Además, está involucrado con el sistema nervioso endocrino e inmunológico al producirse en núcleos hipotalámicos y poblaciones endocrinas cerebrales y extracerebrales y no solo en la médula espinal. El FNC es una neurotrofina y una citocina y también parece estar relacionado con los oncogenes. Con este factor, Levi-Montalcini concluyó que los sistemas nervioso central y periférico no están rígidamente programados y cuestionó el papel real de los tejidos y los órganos periféricos en los centros nerviosos medulares que los inervan.

Robert Provine, uno de sus alumnos y a quien tuve la oportunidad de entrevistar, me compartió que Rita 
Levi-Montalcini fue una de las más creativas, demandantes y luminosas figuras de las ciencias biológicas del siglo XX. Poseía una intuición científica impresionante, una voluntad de hierro, un estricto código ético en el laboratorio, un hábito de trabajo y una disciplina excepcional. Ordenada y tenaz, trabajaba muchas horas al día. No toleraba la estupidez, era temperamental y su estado de ánimo fácil de percibir. Encantadora, educada, de buen humor, hermosa, elegante toda su vida, distinguida, consciente de la moda, con un estilo aristocrático, chic. Provine la describe delgada como modelo, en elegantes trajes sastre o vestidos de cuello alto sin mangas, ensamblados con un saco de gusto exquisito que ella misma diseñaba en seda y brocado italiano; todo combinado con tacones de aguja, el collar de perlas de su madre, una gruesa pulsera de oro y un broche antiguo. Antes de iniciar su clase, le agradaba ponerse una gota de perfume detrás de cada oreja. En palabras de Robert Provine, Rita Levi-Montalcini demostró lo rico que puede ser una existencia longeva en la carrera científica y como la creatividad no tiene edad.

\section{Lecciones}

Ahora, en orden cronológico inverso podemos concluir lo siguiente:

El descubrimiento del FNC es un buen ejemplo de los hallazgos científicos contemporáneos, producto del trabajo de grupos de investigadores apoyados en tecnología sofisticada. Sin embargo, entre esos científicos con frecuencia existe uno que lleva los experimentos y las ideas hasta su última consecuencia. Tal fue el caso de Rita Levi-Montalcini, quien se propuso entender el origen y desarrollo del tejido nervioso en el proceso embrionario y descubrió así el FNC. LeviMontalcini decía que no tenía una inteligencia superior, pero sí una intuición particular en el laboratorio. Al margen de su propia afirmación, es claro que trabajaba intensamente, poseía una magnífica formación, gran disciplina, orden, paciencia, constancia y una enorme resistencia a la adversidad y a la frustración, cualidades que cristalizaron en una vida muy exitosa en el mundo de la investigación científica y en la obtención del Premio Nobel.

Miguel Francisco Jiménez fue un preclaro médico que perteneció a la esfera letrada de su tiempo. Gustó de la docencia, la difusión del conocimiento y, fundamentalmente, del trabajo clínico mediante el cual se advierte la ética y el rigor científico con que trabajaba, así como la importancia que concedía a la experiencia. El cúmulo de años de trabajo le permitió pasar de soluciones prácticas a soluciones epistemológicas ante los problemas de salud que afrontaba. Jiménez llevó a cabo un trabajo científico que enmarca personajes, instituciones, asociaciones académicas, publicaciones y la investigación en sí misma.

Finalmente, si bien muchos de los remedios y las intervenciones citados en la obra de Cervantes para sanar se han quedado en los tiempos de la historia, las mayores lecciones de don Quijote de la Mancha para los médicos de nuestros días serían su bonhomía, filantropía, lealtad, honradez, respeto y tantas virtudes más. En resumen, a través de su loca cordura, habrá que seguir aprendiendo siempre de su amor por los demás.

\section{Agradecimientos}

A la doctora Teresita Corona, presidente de la Academia Nacional de Medicina de México, por la invitación para presentar este simposio.

\section{Conflicto de intereses}

Los autores declaran no tener conflicto de intereses alguno.

\section{Financiamiento}

Los autores no recibieron patrocinio para llevar a cabo este artículo.

\section{Bibliografía}

1. De Cervantes-Saavedra M. Don quijote de la Mancha [edición Guanajuato]. México: Museo Iconográfico del Quijote; 2010.

2. Fernández-del Castillo F. Antología de escritos histórico-médicos del Dr. Francisco Fernández del Castillo. México: Departamento de Historia y Filosofía de la Medicina, Facultad de Medicina, Universidad Nacional Autónoma de México; 1978.

3. Jiménez MF. Discurso pronunciado por D. M. F. Jiménez al comenzar las lecciones de clínica en la Escuela de Medicina. México: Periódico de la Sociedad Filoiátrica de México; 1844.

4. Rodríguez ME. Academia Nacional de Medicina de México. Notas históricas. México: Academia Nacional de Medicina de México/Permanyer; 2018.

5. Laín-Entralgo P. Historia de la medicina. España: Salvat; 1982.

6. Somolinos-D’Ardois G. La obra del Dr. Miguel F. Jiménez. Las conferencias Miguel Jiménez e Ignacio Chávez. México: Academia Nacional de Medicina/Intersistemas; 2015

7. Jiménez MF. Tabardillo. Gac Med Mex. 1864:1:205-216.

8. Jiménez MF. Tratamiento de los abscesos del hígado. Gac Med Mex. 1866;2:6-11.

9. Jiménez MF. Abscesos del hígado. Lecciones de clínica médica. México: La Unión Médica de México; 1857.

10. Levi-Montalcini R. Elogio de la imperfección. España: Ediciones B; 1999.

11. Rodríguez-de Romo AC. ¿Eres exitoso? La historia y los científicos responden. México: Facultad de Medicina, Universidad Nacional Autónoma de México; 2017.

12. Rodríguez-de Romo AC. Chance, creativity and the discovery of the nerve growth factor. J Hist Neurosci. 2007;16:268-287. 